\title{
Clinical validation of eye vergence as an objective marker for diagnosis of Attention Deficit Hyperactivity Disorder in children
}

\author{
Paloma Varela ${ }^{1}$, Flavia Lorena Esposito ${ }^{2,3}$, Imanol Morata ${ }^{3,4}$, Alba Capdevila ${ }^{1}$, Maria Solé Puig ${ }^{2}$, \\ Núria de la Osa ${ }^{5}$, Lourdes Ezpeleta ${ }^{5}$, Stephen V. Faraone ${ }^{6}$, Josep Antoni Ramos-Quiroga ${ }^{7,8}$, Hans \\ Supè̀r, ${ }^{2,3,-11}$ Jose Cañete ${ }^{1}$
}

1. Mental Health Dept, Consorci Sanitari del Maresme, Mataró, Spain

2. Departament de Cognició, Desenvolupament i Psicologia de l'Educació, Faculty of Psychology, University of Barcelona, Barcelona, Spain

3. Braingaze SL, Mataró, Spain

4. Facultat de Matemàtiques i Estadística, B2SLab - Bioinformatics and Biomedical Signals Laboratory Centre de Recerca en enginyeria biomèdica, Universitat Politècnica de Catalunya,

5. Dept Clinical and Health Psychology, Unitat d'Epidemiologia i de Diagnòstic en Psicopatologia del Desenvolupament, Universitat Autònoma de Barcelona (UAB), Bellaterra, Spain

6. Departments of Psychiatry and of Neuroscience and Physiology, Suny Upstate Medical University, Syracuse, NY USA

7. Department of Psychiatry, CIBERSAM, Hospital Universitari Vall d'Hebron, Barcelona, Spain

8. Department of Psychiatry and Forensic Medicine, Universitat Autònoma de Barcelona, Barcelona, Spain

9. Institut de Neurociències, Universitat de Barcelona, Barcelona, Spain

10. Institut de Recerca Pediàtrica Hospital Sant Joan de Déu, Barcelona, Spain

11. ICREA, Barcelona, Spain

Corresponding author: Hans Supèr

Departament de Cognició, Desenvolupament i Psicologia de l’Educació

University of Barcelona (www.ub.edu)

Pg. Vall d'Hebron 171

08035 Barcelona, Spain

Tel: +34933125158

Fax: +34934021363

Skype: h.a.super

Email: hans.super@icrea.cat

\section{Acknowledgements}

We like to thank Prof. dr. E. Baca-Garcia for his helpful comments on a previous version of the manuscript.

Key words: ADHD, Attention deficit hyperactivity disorder, Eye vergence, Diagnosis, Children, Biomarker, Binocular. 
Objective: Attention deficit hyperactivity disorder (ADHD) is a neurodevelopmental disorder diagnosed in 3-7\% of children. Apart from symptoms of hyperactivity, impulsivity and inattention, ADHD youth show poor oculomotor control. Of particular interest is eye vergence, i.e. the movement of both eyes in opposite direction. Recent research shows that eyes converge when orienting visual attention and that such attention related eye vergence is weak or absent in ADHD children. These findings lead to the hypothesis that vergence responses can be used as a marker to detect ADHD. Method: To validate eye vergence as a marker to classify ADHD, we assessed the modulation in the angle of vergence of children $(N=43)$ previously diagnosed with ADHD while performing an attention task and compared the results to age-matched clinical controls $(\mathrm{N}=19)$ and healthy peers $(\mathrm{N}=30)$. Results: We observed relatively strong vergence responses in healthy subjects and weak vergence in the clinical control group. Children with ADHD showed no significant modulation in the angle of eye vergence. Based on these results, machine learning models correctly classified ADHD patients $(\mathrm{N}=21)$ from healthy controls $(\mathrm{N}=21)$ with an accuracy of $96.3 \%$ (FP: $5.12 \%$; $F N$ : 0\%; AUC: 0.99$)$ and ADHD children ( $N=11)$ from clinical controls ( $N=14)$ with an accuracy of $85.7 \%$ (FP: 4.5\%; FN: 19.2\%, AUC: 0.90). Conclusion: Our work shows that, in combination with an attention task eye vergence responses can be used as an objective marker to detect ADHD in children.

Trial Registration: AEMPS, no expte. 548/15/EC. 
Attention deficit hyperactivity disorder (ADHD) is one of a common neurodevelopmental disorder affecting 3 to $7 \%$ of school-aged children worldwide. It is characterized by a low degree of attention, a high degree of hyperactivity and impulsivity, and the inability to inhibit inappropriate actions.

The altered behavior of ADHD patients is not limited to general conduct but it is also observed at the level of saccadic eye movement behavior. ADHD patients have more difficulty suppressing saccadic eye movements when fixation is required (Karatekin and Asarnow, 1999; Munoz et al., 2003) and the rate of micro-saccades is reported to be higher in ADHD subjects compared to controls, especially in the time intervals around stimulus onset (Fried et al., 2014) and after cue presentation, although suppression has also been reported (Engbert and Kliegl, 2003). The poor eye control of ADHD patients is furthermore expressed as an increased variability in the latencies of saccadic responses to visual stimuli compared to healthy controls (Leth-Steensen et al., 2001, Kuntsi et al., 2006). Poor control of saccadic behavior has been observed in anti-saccades (Leth-Steensen et al., 2001), visually guided saccades (Leth-Steensen et al., 2001; Mostofsky et al., 2001; Munoz et al., 2003), and memory guided saccades (Rommelse et al., 2008). Moreover whereas healthy subjects show an asymmetry in eye movement control where eyes move faster when controlled by the right cerebral hemisphere, ADHD children do not show this asymmetry (Rothlind et al., 1991). In contrast to fast saccadic eye movements, slower smooth pursuit eye movements appear to be within normal range in ADHD patients (Ross et al., 2000).

Besides the saccadic eye movements, dis-conjugate eye movements or vergence, i.e. where the eyes move in opposite direction (Fig. 1) are affected in ADHD patients (Solé Puig et al., 2015). This finding is of relevance as recent data provide evidence showing that eyes converge during orienting attention (Solé Puig et al., 2013a,b). During gaze fixation the eyes briefly converge after the presentation of a stimulus, which indicates the location of an upcoming peripheral target but not or weakly after a stimulus that was not informative about the location of the peripheral target (Solé Puig et al., 2013a). The strength and timing of eye vergence correlate with the onset and strength of the visual event related potentials (VERPs) at parietal locations (Supèr et al., 2014). Also stimulus contrast is associated with strength of convergence (Sole et al., 2013a) where high stimulus contrast relates to larger modulation of the angle of eye vergence. Moreover, 
detected targets were accompanied by eye vergence, whereas targets, which were not detected, were not (Sole et al 2013a).

The diagnosis of ADHD is clinical based upon criteria established by classification systems such as the DSM-V. Even though the clinical diagnosis shows considerable levels of concurrent and predictive validity (Faraone, 2005; Faraone et al., 2000) concerns persist, and additional tools are needed to support ADHD diagnosis. The observation that attention-related eye vergence is poorly present in children with ADHD (Solé Puig et al., 2015) led to the idea that evaluating eye vergence during an attention task can be used as an objective measure to support the clinical diagnosis of ADHD. A recent study reporting abnormalities in the brain structure, which control eye vergence, in ADHD patients supported this notion (Johnton et al., 2014). In fact, in a preliminary report we were able to classify ADHD in children using vergence responses (Lorena et al., 2016). The aim of the present study was, therefore, replicate our earlier findings and to validate eye vergence as a marker tool for classifying ADHD. We assessed the modulation of the angle of vergence in children previously diagnosed with ADHD while performing a child friendly attention task and compared the results to the responses from age-matched clinical controls and healthy subjects.

\section{Methods}

\section{Participants}

Forty-three children aged between 7 and 17 years (mean \pm std: $11.95 \pm 3.06$ ) diagnosed with ADHD, and 19 clinical controls (mean \pm std: $11.57 \pm 2.86$ ) participated in the study. Clinical controls were children referred to the hospital for attentional and/or conduct problems but after first clinical assessment diagnosed as not having ADHD but suffering other mental problems. None of the participants were taking medication for ADHD. Patients were recruited through the Child and Adolescent Health Mental Center from the Hospital Mataro of the Consorci Sanitari del Maresme. The subjects $(\mathrm{N}=30)$ from the control group were sex/age matched healthy children showing no attention or conduct problems (mean \pm std: $8.85 \pm 0.49$ years) recruited via a public school. In addition, a separate population of 67 children ( 21 healthy controls; 14 clinical controls, 
and 32 ADHD children) were used for validation purposes. All the clinical diagnoses of ADHD were made by clinical psychiatrists. All cases were diagnosed using the American Psychiatric Association's Diagnostic and Statistical Manual-IV-Text Revision criteria including a psychiatric and psychologist interview to assess the presence of symptoms of inattention, hyperactivity and impulsivity during the last 6 months. Also the beginning of the symptoms before 7 years of age and the persistence of clinical dysfunction in at least two settings (school and home) were used as criteria. Furthermore, we analyzed psychopathology and comorbidity using Kiddie Schedule for Affective Disorders and Schizophrenia for School-Age ChildrenPresent and Lifetime version (K-SADS-PL; Kaufman et al., 1997). Part of the medical examination and psychiatric evaluation of all patients for diagnosing ADHD was the inquiry about visual problems. The survey included specific questions on strabismus and accommodation insufficiency. All participants had normal or corrected-to-normal visual acuity.

\section{Inclusion and exclusion criteria}

The inclusion criteria were: (1) between 7 and 17 years of age; (2) a diagnosis of ADHD without mental retardation; (3) Spanish mother tongue or fluency in Spanish; and (4) informed consent for the study signed by the parent or legal guardian with patient assent. The exclusion criteria were: (1) a history of head injury with loss of consciousness or other neurological illness (2) mental retardation or other significant disorders like a pervasive developmental disorder (3) Visual or auditory problems.

\section{Ethics statement}

Before participating in the study, written informed consent from the parents on behalf of the children enrolled in our study was obtained in accordance with the Helsinki Declaration. The study was approved by the Ethics Committee of the University of Barcelona and of Consorci Sanitari del Maresme, and the study was registered at AEMPS (identifier: 548/15/EC).

\section{Apparatus}


We used the BGaze system (Braingaze SL, Mataró, Spain) for presenting the visual stimuli synced with a remote eye tracker. The BGaze system includes a $30 \mathrm{~Hz}$ binocular eye tracker (X2-30, Tobii Technology AB, Sweden). The display resolution was 1024 x 768 pixels.

\section{Procedure}

Children sat in a dimly lit room of the hospital or school, in front of the PC monitor at a distance of $55 \mathrm{~cm}$. During the entire task a chinrest was used to prevent head movements. The eye tracking equipment was calibrated ( 5 points, binocular) for each participant at the beginning of the experiment by BGaze eye tracking software. Before starting the task, all children practiced with cue and no-cue trials (20 trials) to become familiar with the task. The entire procedure took about 12 minutes to complete. After testing, the saved behavioral and eye data was stored and assigned a random number. Validation was done double blinded (Fig 2).

\section{Visual cue experiment}

To assess orienting visual attention we used a paradigm in which the children were required to discriminate cartoon images of a tadpole from a fish (Fig. 3). Each trial started with the presentation of a central frog (size of approx. $3 \times 4^{0}$ ) with his eyes closed, together with two small pool on either side (size of $2 \times 3^{0}$; eccentricity of 60 ). After $500 \mathrm{~ms}$ fixating, the frog opened the eyes looking towards the left or right side, or straight ahead. In the former case the frog's gaze served as a cue to inform the child about the location (left or right) of the upcoming image of a tadpole or fish (informative cue condition). In the latter case the child was unaware of the stimulus location (no-informative cue condition). In total there were 128 trials. Fifty percent of the trials contained an informative cue and trials with different cue conditions were randomly interleaved. The fish or tadpole cartoon was presented for $1500 \mathrm{~ms}$. During the trial the child was required to maintain fixation at the central frog image, and had to respond by pressing a button when a tadpole was presented and refrain from responding when a fish appeared. When the child correctly identified a tadpole feedback was given by a small jump of the frog. The next trial started automatically at the end of a trial. 


\section{Data analysis}

In total there were 3354 (1661 no-informative cue trials and 1693 informative cue trials) trials in the healthy control group, and 2240 (1133 no-informative cue trials and 1107 informative cue trials) in the clinical group, and 5036 ( 2537 no-informative cue trials and 2499 informative cue trials) in the ADHD group. The angle of eye vergence was calculated using the cross product of both gaze vectors. Gaze vectors correspond to the lines between the $3 \mathrm{D}$ eye positions and 2D gaze positions in a common coordinate system. Samples that gave a low validity score according to the Tobii eye tracker software were set as missing. In total, 10$20 \%$ of the samples were invalid. Low validity scores usually happen during saccades and blinks. From the remaining samples we calculated the point-wise median of all trials for conditions and groups separately. We chose to use the median instead of the mean in order to mitigate the effect of occasional outliers and thereby reduce bias. To reduce irregularities the obtained signal was then smoothed using a moving median and thereafter a moving average with a $200 \mathrm{~ms}$ window. For statistical analysis, we used bootstrapping and permutation analysis in order to simulate point-wise the distributions of the medians.

For classification purposes we used machine learning algorithms (Fig. 2). The classification model consists of two layers. In the first layer, a Radial Basis Function SVM model (gamma $=6.5$ ) was trained and tested to separate healthy children from ADHD children using a set of three vergence features extracted from no-cue, left-cue and right-cue signals. In the second layer, two nearest-neighbors models (1-NN and 3-NN) were used to distinguish between clinical controls and ADHD children. In the second layer, overall vergence level, vergence variations in velocity were used as features. For each layer a 30-fold stratified cross-validation routine using an 80-20 randomized split was applied. The validation was done with an unseen population. Only subjects that were not classified as healthy controls and the ones that reached poor confidence levels in the first layer entered the second layer. The final classification was based on the label that gave the highest confidence level. 


\section{Results}

\section{Behavior}

The overall behavioral reaction times to target stimuli (tadpole images) in the informative cue condition were similar for all groups (mean \pm std; controls: $678.8 \pm 231.1 \mathrm{~ms}$; clinical controls: $644.3 \pm 200.8 \mathrm{~ms}$; ADHD: $664.1 \pm 248.8 \mathrm{~ms}$; Tukey's rank test: all possible combinations: $p>0.05)$. In the no-informative cue condition, children on average responded slower to target stimuli than in the informative cue condition. Healthy controls responded (mean \pm std; $712.6 \pm 210.6 \mathrm{~ms})$ significantly $(p<0.01)$ slower than the children from the other two groups (mean \pm std; clinical controls: $661.7 \pm 194.6 \mathrm{~ms}$; ADHD: $683.3 \pm 240.5 \mathrm{~ms}$ ). Between clinical controls and ADHD children no significant differences were observed in reaction times. The variability in reaction times were different between the three groups $(p<0.01)$.

For healthy controls, the hit rates for targets were $81.7 \%$ and $81.7 \%$ and the correct rejection rates for the distractors was $77.3 \%$ and $75.9 \%$ in the informative and no-informative cue conditions, respectively. In the clinical control group, the hit rates were $60.0 \%$ and $64.2 \%$, and the correct rejection rates were $64.3 \%$, and $62.2 \%$ in the informative and no-informative cue conditions, respectively. In the ADHD group, hit rates were $66.1 \%$ and $67.8 \%$, and correct rejection rates were $67.6 \%$ and $67.6 \%$ in the informative and noinformative cue conditions, respectively. The differences in the hit rates between healthy controls and the clinical populations were statistically significant in both cue conditions (Tukey's rank test: all: $p<0.05)$. The differences in the correct rejection rates were not significant (Tukey's rank test: all possible combinations: $\mathrm{p}>0.1)$.

\section{Eye vergence data}

During the trials, the angle of eye vergence was not constant even though subjects maintained gaze fixation at the central image. Notably at the end of the trial during the stimulus period (when the target/distractor is presented) the angle of eye vergence decreased, which means that the eyes diverged (Figs. 4). Around $600 \mathrm{~ms}$ after the onset of the target/distractor, the angle of eye vergence reached a minimum and returned to the initial baseline level. The changes in the angle of eye vergence are observed in trials belonging to the 
informative cue and no-informative cue conditions but they are more pronounced in the former condition. In contrast to the results obtained from healthy subjects, the results from the clinical groups show remarkably less modulation in the angle of eye vergence. Especially, children of the ADHD group showed little to no changes in the angle of eye vergence during the task.

\section{Cue versus no-cue vergence}

In the healthy and the clinical control groups, eye vergence responses are stronger (i.e. the angle of eye vergence is larger meaning that the eyes converge), during the informative cue period compared to the vergence responses during the no-informative cue period (Fig. 5). In the ADHD group no difference in vergence responses during the cue period is observed (Fig 5).

\section{Distractor versus target}

To appreciate the relation of eye vergence to stimulus relevance we compared the vergence responses to targets (tadpole images) to the responses to distractors (fish images). In the healthy control group the angle of eye vergence decreased equally strongly during the stimulus period in both cases (Fig. 6). In the clinical control group, eye vergence to distractors was as strong as in the healthy control group but was it was less noticeable in the target condition (Fig 6). In the ADHD group both for targets and distractors no changes in the angle of eye vergence were observed.

\section{Classification}

By applying machine learning algorithms, we assessed whether using features of the modulation in the angle of eye vergence we were able to discriminate ADHD subjects from the healthy and clinical controls. We used a three-step classification procedure (Fig. 2). In the cross-validation stages we obtained accuracy of $96 \%$ in layer 1 and $88 \%$ in layer 2 . In the validation stage with unseen subjects, the results show (Fig. 7) that with a $96.3 \%$ precision (AUC 0.99) ADHD subjects can be separated from healthy subjects. The false positive rate, i.e. healthy patient diagnosed as $A D H D$, was $5.12 \%$ and the false negative rate, i.e. ADHD 
patients classified as healthy was $0 \%$. The separation between clinical groups reached an accuracy of $85.7 \%$ with an AUC of 0.90 . False positive and false negative rates were $4.5 \%$ and $19.23 \%$, respectively. Since behavioral performance data showed statistical significance, we added behavioral features (average response time, variability in response time, hit rate and correct rejection rate) to the models in order improving accuracy. Instead of improvement reduced accuracies were obtained. Neither adding pupil size data to the model improved classification outcomes.

\section{Pupil diameter}

Neural circuits for controlling eye vergence partly overlap with those that control pupil size. To see if the size of the pupil changes during the attention task, we calculated pupil diameter. The results show that the diameter of the pupil increases during the task. This increase was stronger in the healthy control group than in the clinical groups. There was no significant difference in pupil diameter between the clinical groups, except in the no-informative cue condition where the clinical control group showed a stronger increase in pupil diameter compared to the pupil changes of the ADHD group (Fig. 8). Because healthy subjects showed different pupil modulations, we tried to classify ADHD children based on pupil size. However, classification in the validation stage gave poor results with maximal AUCs of 0.6 (Fig. 9).

\section{Behavioral and vergence responses}

Previous research has shown an absence of vergence responses when subjects fail to detect a stimulus (Solé Puig et al., 2013a). To know whether healthy and clinical groups differ we compared the vergence responses to the behavioral responses (Fig. 10). For correct responses to targets (hits) a strong vergence response in the healthy and clinical control groups was noticed. In contrast, when subjects fail to respond to targets (misses) a clear modulation in the vergence angle was absent. For correct rejections and false alarms healthy subjects showed vergence responses but no or weak modulation in the angle of eye vergence was seen in the clinical groups. 
We next aligned the vergence responses at onset of the behavioral response, i.e. when subject pressed the response button (Fig. 11). In the healthy control group there was a clear dip in the vergenec responses centered on the behavioral response. This was also true for the clinical control group in the noinformative cue condition and for responses to distractors (false alarms). No clear response dip was observed in the informative cue condition and for hit trials. ADHD subjects showed no response modulation around behavioral response onsets.

\section{Task duration}

During the 12 minute task, subjects may become fatigued or bored by stimulus repetition, especially those in the ADHD group as they have difficulties remaining focused. To assess the possible influence on vergence responses, we compared the modulation in the average vergence angle during the first half of the task and compared that to the responses of the second half of the task. There is a slightly lower modulation in the first half during the stimulus period in the healthy group and during the cue period in the ADHD group (Fig. 12). In the clinical control group no difference was seen.

\section{Stimulus laterality}

Many studies report compromised brain lateralization in patients with ADHD. We therefore tested whether vergence responses show such laterality effect. We analyzed vergence to stimuli presented on the left and right sides separately. In none of the groups was there a clear difference in the modulation in the angle of eye vergence between left and right conditions (Fig. 13).

\section{Discussion}

In this study we tested eye vergence modulation while performing an attention task as a tool to discriminate ADHD from non-ADHD children. We applied a child friendly attention task for ten to twelve minutes and recorded with a remote eye tracker eye position data from which vergence was calculated. The principal 
finding is that children belonging to the healthy control group showed clear modulation in the angle of eye vergence whereas children in the clinical groups showed weak to no significant modulation in the angle of eye vergence while performing the attention task. The angle of eye vergence decreased during the stimulus period when the target/distractor stimulus was presented. This signifies that the eyes diverged. In addition, the vergence angle from the healthy control group was larger in the informative cue condition than in the no-informative cue condition during the period of cue presentation. These findings agree with our earlier observations of attention-related eye vergence in children (Solé Puig et al., 2013a). Furthermore, we show modest vergence responses in a clinical control group. Even though children of the clinical control group showed, in general, weaker modulation in the angle of eye vergence, there was a small significant difference in vergence between the cue conditions. Children from the ADHD group however showed weak vergence responses and no difference in vergence between cue conditions was observed. Thus ADHD children appear less sensitive to visual stimulation but they are also vulnerable when orienting attention is required.

The current results confirms our previous findings showing poor attention related eye vergence in ADHD children (Solé Puig et al., 2015) and that vergence can be a used to discriminate ADHD in children (Lorena et al., 2016). In the latter study we evaluated 4 classes of supervised machine learning classifiers (in total 138 different models) with a validation set of 232 children of a school cohort. The average accuracy of these models was $90 \%$ (minimum $86.21 \%$; maximum, $95.26 \%$ ). In the current study we applied a twostep classifier. This was necessary because we included a clinical cohort, which compared to healthy controls gave more subtle differences in the angle of eye vergence.

The overall weak modulation in the angle of eye vergence in ADHD children may indicate that the vergence system is less sensitive to visual stimulation. This may explain the absence of clear vergence responses in healthy controls when failing to detect the target (Solé Puig et al., 2013a; current study). The absence of a difference between vergence responses from the informative and no-informative cue condition suggests that attentional control of eye vergence by presumably higher cortical areas is impaired or the vergence system is still immature (Kirby et al., 20011; Bucci et al., 2012; Prado et al., 2007). 


\section{Markers of ADHD}

The clinical diagnosis of ADHD shows considerable levels of concurrent and predictive validity (Faraone, 2005; Faraone et al., 2000). Nevertheless, concerns about diagnostic accuracy persist. The diagnosis has been called "subjective" because it relies on clinician evaluation of responses from patients, parents and/or informants. Some suggest that the use of subjective diagnostic procedures leads to the over-diagnosis of ADHD (Bruchmuller et al., 2012, Visser et al., 2014) while other studies have raised concerns about the under-diagnosis of ADHD. In response to such concerns, researchers have sought to develop objective measures to diagnose ADHD.

Much research has examined peripheral biochemical markers. Meta-analyses of these studies indicate that five measures differentiated ADHD and control patients (Norepinephrine (NE), 3-Methoxy-4hydroxyphenylethylene glycol (MHPG), monoamine oxidase (MAO), zinc and cortisol) (Faraone et al., 2014; Scassellati et al., 2012). Moreover, NE, MHPG, MAO, b-phenylethylamine and cortisol were responsive to ADHD medications. Meta-analysis also shows that peripheral measures of oxidative stress differ between ADHD and control subjects (Joseph et al., 2015). Other approaches to biomarker development for ADHD have used neuropsychological (Ritsner, 2009), electroencephalographic (Snyder et al., 2015), actigraphy (Dane et al., 2000), structural imaging (Silk et al., 2009) and functional imaging (Bush et al., 2005) methods. Continuous performance tests (CPTs) (e.g. Homack and Riccio, 2006; Riccio and Reynolds, 2001; Corkum and Siegel, 1993) have been evaluated in many studies.

Most methods that are currently being used to support the clinical diagnosis quantify symptoms of ADHD. The AULA Nesplora (AULA) is a continuous performance test (CPT) that shows clear correlations with the Conners CPT (Díaz-Oruate et al., 2014). AULA reports > 90\% accuracy at their company website, and may be useful in establishing a differential ADHD diagnosis (Areces et al., 2016). Quotient reports a crossvalidated AUC of 0.716 (unpublished data at company web site) based on comparing ADHD patients with healthy controls. They report higher ROCs that have not been cross-validated. The ability of the QbTest to identify ADHD in children is moderate with sensitivity ranging from $47 \%$ to $67 \%$ and specificity from $72 \%$ to 84\% (Hult et al., 2015; Reh et al., 2015). The adult version of the QbTest gives similar results with an overall 
correct classification of 72.1\% (Edebol, et al., 2011, 2013; Söderström et al., 2014). Measuring body movements with wireless inertial sensors gives a classification of $>95 \%$ (O'Mahonya et al., 2014) and classification based on micro-saccades yields an accuracy of 70\% (sensitivity: $59 \%$; specificity: $82 \%$; Fried et al., 2014). The Neuropsychiatric EEG-Based Assessment Aid (NEBA) is an EEG brainwave test for ADHD based on theta/beta ratio (TBR). Integration of NEBA outcomes with a clinician's ADHD evaluations improves diagnostic accuracy from $61 \%$ to $88 \%$ (Snyder et al., 2015). A study examining the TBR in ADHD and normal children reported a sensitivity of 0.86 and a specificity 0.57 (Sangal and Sangal, 2015).

So far, no method has shown sufficient sensitivity and specificity when predicting "gold standard" clinical diagnoses of the disorder. According to the task force of the World Federation of ADHD (WF-ADHD) a useful biomarker needs to meet the following criteria (Thome et al., 2012): sensitivity exceeding $80 \%$, specificity exceeding $80 \%$, the putative biomarker is reliable, reproducible, inexpensive, non-invasive, easy to use, and has been confirmed by at least two independent studies. The use of eye vergence recordings to classify ADHD meets all of the WF-ADHD criteria but needs to be confirmed by additional studies.

\section{Binocular vision difficulties in ADHD}

The finding of altered vergence in ADHD is not surprising given that binocular vision in ADHD children is impaired. Convergence insufficiency $(\mathrm{Cl})$, which is a common binocular disorder characterized by the inability to obtain a single visual field while working at a near distance (Rouse et al., 1999; Borsting et al., 2003, 2005, 2011), is prevalent in children with ADHD (Granet et al., 2005), and has been shown to relate to attention problems (Borsting et al., 2003; Rouse et al., 2009; Poltavski et al., 2012). The primary source of $\mathrm{Cl}$ symptoms may be accommodative insufficiency (Marran et al., 2006) and thus occurs at close distances. In our study the target distances fell well outside the range of distances of $\mathrm{Cl}$ for children. This means that our observed disruption in vergence modulation does not reflect $\mathrm{Cl}$ but represents a novel role of eye vergence in visual attention (Solé Puig et al. 2013a,b). 
Top down attention originates in the frontal cortex (Bisley, 2011; Thompson et al., 2005) and reduced or distorted activation in prefrontal regions of ADHD patients has been observed (Di Michele et al., 2005; Kraina and Castellanos, 2006; Valera et al., 2007; Bush, 2011; Soliva, 2011; Arnsten and Rubia, 2006; de Zeeuw et al., 2013). The frontal cortex controls eye vergence (Gamlin and Yoon, 2000) and may be the source of our observed attention related vergence. Thus reduced functionality of the frontal cortex in ADHD patients may produce a distorted modulation in attention related vergence. The frontal cortex is connected to the reticular formation in the brainstem, where premotor neurons reside that control eye vergence (Chaturvedi and Van Gisbergen, 2000; Mays, 1984; Judge and Cumming, 1986; Gamlin, 2002; Suzuki et al., 2004; Coubard, 2013). The reticular formation of the brainstem forms part of a broader pathway, including the frontal and parietal regions of the cerebral cortex (Gnadt and Mays, 1995; Gamlin and Yoon, 2000; Alvarez et al., 2014) and cerebellum (Alvarez et al., 2014; Versino et al., 1996; Nitta et al., 2008). These structures, which form part of the attention system of the brain also are involved in the control of vergence. This suggests that the neural circuits controlling vergence and attention are closely linked.

\section{Study limitations and future research}

Together with our earlier study (Solé Puig et al., 2015), this is the second study validating attention related vergence in children as an objective marker for ADHD diagnosis. ADHD is multifaceted pathology where patients show various cognitive deviations. Our current study assessed only orienting attention and therefore more testing is desirable using different cognitive tasks. The ability of eye vergence to classify ADHD presentation "specifiers" (subtypes) and the effect of medication on vergence responses needs to be investigated in future studies. The findings of disrupted eye vergence in ADHD children agrees with the general impression of poor binocular control in children with attention problems. However, further studies are needed to assess role of eye vergence in attention.

\section{Conclusions}


Our observations show that attention related vergence differs between healthy controls, clinical controls, and children with ADHD. Solely based on features of the modulation in the angle of eye vergence, we were able to classify ADHD from healthy and clinical control subjects with high precision. The results therefore clearly demonstrate that assessment of vergence during a child friendly attention task is a useful, observer independent tool supporting clinical diagnosis of child ADHD.

\section{Acknowledgements}

We like to thank Prof. dr. E. Baca-Garcia for his helpful comments on a previous version of the manuscript.

\section{Potential conflicts of interest}

Flavia Lorena Esposito and Imanol Morata are employees of Braingaze. Hans Supèr is co-founder of Braingaze.

\section{Funding/support}

This research was supported by Industrial Doctorates grants to Flavia Lorena Esposito and Imanol Morata from AGAUR and financial support from CDTI, Spain to Braingaze.

\section{References}

Alvarez TL, Jaswal R, Gohel S, Biswal BB (2014) Functional activity within the frontal eye fields, posterior parietal cortex, and cerebellar vermis significantly correlates to symmetrical vergence peak velocity: an ROIbased, fMRI study of vergence training. Front Integr Neurosci. 8:50. doi: 10.3389/fnint.2014.00050.

Areces D, Rodríguez C, García T, Cueli M, González-Castro P (2016) Efficacy of a Continuous Performance Test Based on Virtual Reality in the Diagnosis of ADHD and Its Clinical Presentations. J Atten Disord. pii: 1087054716629711. 
Arnsten AF, Rubia K. (2006) Neurobiological circuits regulating attention, cognitive control, motivation, and emotion: disruptions in neurodevelopmental psychiatric disorders. Visual and oculomotor selection: links, causes and implications for spatial attention. Trends in Cognitive Science 10: 124-130

Bisley, JW. (2011) The neural basis of visual attention. Journal Physiology, 589: 49-57.

Borsting EJ, Rouse MW, Mitchell GL, Scheiman M, Cotter SA, Cooper J, Kulp MT, London R. (2003) Validity and reliability of the revised convergence insufficiency symptom survey in children aged 9 to 18 years. Optometry Vision Science. 80: 832-838.

Borsting E, Rouse M, Chu R. (2005) Measuring ADHD behaviors in children with symptomatic accommodative dysfunction or convergence insufficiency: a preliminary study. Optometry 76: 588-5892.

Borsting E, Mitchell GL, Scheiman M, Chase C, Kulp M, Cotter S. (2011) CITT-RS Study Group. Improvement in academic behaviors and attention following successful treatment of convergence insufficiency. Optometry Vision Science 88, E-abstract 110567.

Bruchmuller K, Margraf J, Schneider S. (2012) Is ADHD diagnosed in accord with diagnostic criteria? Overdiagnosis and influence of client gender on diagnosis. J Consult Clin Psychol. 80(1):128-38.

Bucci MP, Nassibi N, Gerard CL, Bui-Quoc E, Seassau M (2012) Immaturity of the oculomotor saccade and vergence interaction in dyslexic children: evidence from a reading and visual search study. PLoS One. 7(3):e33458.

Bush G. (2011) Cingulate, frontal, and parietal cortical dysfunction in attention-deficit/ hyperactivity disorder. Biological Psychiatry 201:69(12): 1160-1167. 
Bush G, Valera EM, Seidman LJ. (2005) Functional neuroimaging of attention-deficit/hyperactivity disorder: a review and suggested future directions. Biol Psychiatry. 57(11):1273-84.

Chaturvedi V, Van Gisbergen JA (2000) Stimulation in the rostral pole of monkey superior colliculus: effects on vergence eye movements. Exp Brain Res. 32(1):72-8.

Corkum PV, Siegel LS. (1993) Is the Continuous Performance Task a valuable research tool for use with children with Attention-Deficit-Hyperactivity Disorder? J Child Psychol Psychiatry. 34(7):1217-39.

Coubard OA (2013) Saccade and vergence eye movements: a review of motor and premotor commands. Eur J Neurosci., 38(10):3384-97. doi: 10.1111/ejn.12356.

Dane AV, Schachar RJ, Tannock R. (2000) Does actigraphy differentiate ADHD subtypes in a clinical research setting? Journal of the American Academy of Child and Adolescent Psychiatry 39(6):752-60.

Delgado-Mejía ID, Palencia-Avendaño ML, Mogollón-Rincón C, Etchepareborda MC. (2014) Theta/beta ratio (NEBA) in the diagnosis of attention deficit hyperactivity disorder. Rev Neurol. 2014 Feb 24;58 Suppl 1:S57-63.

De Zeeuw P. Mandl RC, Hulshoff Pol HE, van Engeland H, Durston S. (2013) Decreased frontostriatal microstructural organization in attention deficit/hyperactivity disorder. Human Brain Mapping 33(8): 19411951. 
Díaz-Orueta U, Garcia-López C, Crespo-Eguílaz N, Sánchez-Carpintero R, Climent G, Narbona J. (2014) AULA virtual reality test as an attention measure: convergent validity with Conners' Continuous Performance Test. Child Neuropsychol. (3):328-42. doi: 10.1080/ 09297049.2013.792332.

Di Michele F, Prichep L, John ER, Chabot RJ. (2005) The neurophysiology of attention-deficit/hyperactivity disorder. International Journal Psychophysiology 58(1): 81-93.

Engbert R, Kliegl R. (2003) Microsaccades uncover the orientation of covert attention. Vision Research 43: $1035-1045$.

Edebol H, Helldin L, Norlander T. (2013) Measuring adult Attention Deficit Hyperactivity Disorder using the Quantified Behavior Test Plus. Psych J. 2013 Apr; 2(1): 48-62.

Edebol H, Helldin L, Holmberg E, Gustafsson S (2011) In search for objective measures of hyperactivity, impulsivity and inattention in adult attention deficit hyperactivity disorder using the Quantified Behaviour Test Plus. Europe's Journal of Psychology, Vol. 7, no 3, 443-457

Faraone SV. (2005) The scientific foundation for understanding attention-deficit/hyperactivity disorder as a valid psychiatric disorder. Eur Child Adolesc Psychiatry 14:1-10.

Faraone SV, Biederman J, Spencer T, Wilens T, Seidman L, Mick E, et al (2000) Attention deficit hyperactivity disorder in adults: an overview. Biological psychiatry 48(1):9-20.

Faraone SV, Bonvicini C, Scassellati C. (2014) Biomarkers in the diagnosis of ADHD--promising directions. Curr Psychiatry Rep. 16(11):497. 
Fried M, Tsitsiashvili E, Bonneh YS, Sterkin A, Wygnanski-Jaffe T, Epstein T, Polat U (2014) ADHD subjects fail to suppress eye blinks and microsaccades while anticipating visual stimuli but recover with medication, Vision Research, 101:62-72.

Gamlin PD, Yoon K (2000) An area for vergence eye movement in primate frontal cortex. Nature 407:10031007.

Gamlin PD (2002) Neural Mechanisms for the Control of Vergence Eye Movements. Annals of the New York Academy of Sciences, 956:264-272.

Ginsberg Y, Quintero J, Anand E, Casillas M, Upadhyaya HP. (2014) Underdiagnosis of attentiondeficit/hyperactivity disorder in adult patients: a review of the literature. Prim Care Companion CNS Disord. $16(3)$.

Gnadt JW, Mays LE (1995) Neurons in monkey parietal area LIP are tuned for eye-movement parameters in three-dimensional space. J Neurophysiol. 73:280-97.

Granet DB, Gomi CF, Ventura R. Miller-Scholte A. (2005) The relationship between convergence insufficiency and ADHD. Strabismus 13: 163-168.

Homack S, Riccio CA. (2006) Conners' Continuous Performance Test (2nd ed.; CCPT-II). J Atten Disord. 9(3):556-8.

Hult N, Kadesjö J, Kadesjö B, Gillberg C, Billstedt E (2015) ADHD and the QbTest: Diagnostic Validity of QbTest. J Atten Disord pii: 1087054715595697. 
Johnton BA, Mwangi B, Matthews K, Coghill D, Konrda K, Steele JD. (2014) Brainstem abnormalities in Attention Deficit Hyperactivity Disorder support high accuracy individual diagnostic classification. Human Brain Mapping 35: 5179-5189.

Joseph N, Zhang-James Y, Perl A, Faraone SV. (2015) Oxidative Stress and Attention Deficit Hyperactivity Disorder: A Meta-Analysis J Atten Disord. 19(11):915-24.

Judge SJ, Cumming BG. (1986) Neurons in the monkey midbrain with activity related to vergence eye movement and accommodation. J Neurophysiol. 55:915-930.

Kaufman J, Birmaher B, Brent D, Rao U, Flynn C, Moreci P Williamson D, Ryan N. (1997) Schedule for Affective Disorders and Schizophre- nia for School-Age Children-Present and lifetime version (K-SADS-PL): Initial reliability and validity data. Journal of the American Academy of Child and Adolescent Psychiatry, 36 (7), 980-988

Karatekin C and Asarnow RF (1999) ADHD children showed a trend toward shorter fixations than normals on the question requiring the most detailed analysis. Exploratory Eye Movements to Pictures in ChildhoodOnset Schizophrenia and Attention-Deficit/Hyperactivity Disorder (ADHD) Journal of Abnormal Child Psychology, Vol. 27, Issue 1, pp 35-49

Kirkby, J.A., Blythe, H.I., Drieghe, D., \& Liversedge, S.P. (2011) Reading text increases binocular disparity in dyslexic children. PLoS One, 6 (11): e27105.

Kraina A, Castellanos FX. (2006 ) Brain development and ADHD. Clinical Psychology Review 26(4): 433-444. 
Kuntsi J, Rogers H, Swinard G, Börger N, van der Meere J, Rijsdijk F, Asherson P. Reaction time, inhibition, working memory and 'delay aversion' performance: genetic influences and their interpretation. Psychological Medicine 2006: 36(11): 1613-1624.

Leth-Steensen C, Elbaz ZK, Douglas VI. (2000) Mean response times, variability, and skew in the responding of ADHD children: a response time distributional approach. Acta Psychology 104(2): 167-190.

Lorena Esposito F, Varela P, Ozan Alkan E, Morata I, de la Osa N, Ezpeleta L, Cañete Crespillo J, Supèr H (2016) Validation of BGaze method supporting ADHD diagnosis European Psychiatry 33, S135

Marran LF, De Land PN, Nguyen AL. (2006) Accommodative insufficiency is the primary source of symptoms in children diagnosed with convergence insufficiency. Optometry Vision Science 83(5): 281-289.

Mays LE (1984). Neural control of vergence eye movements: convergence and divergence neurons in midbrain. J Neurophysiol. 51:1091-1108.

Mostofsky SH, Lasker AG, Cutting LE, Denckla MB, Zee, DS. (2001) Oculomotor abnormalities in attention deficit hyperactivity disorder: a preliminary study. Neurology 57(3): 423-430.

Munoz DP, Armstrong IT, Hampton KA, Moore KD (2003) Altered Control of Visual Fixation and Saccadic Eye Movements in Attention-Deficit Hyperactivity Disorder. Journal of Neurophysiology 90 (1) 503 514; DOI:10.1152/jn.00192.2003

NICE Clinical Guidelines. The British Psychological Society and The Royal College of Psychiatrists. Diagnosis and management of ADHD in children, young people and adults. 2009. National Clinical Practice Guideline Number 72. National Collaborating Centre for Mental Health. London. 
Nitta T, Akao T, Kurkin S, Fukushima K (2008) Vergence eye movement signals in the cerebellar dorsal vermis. Prog Brain Res. 171:173-6. doi: 10.1016/S0079-6123(08)00623-7.

O’Mahonya N, Florentino-Liano B, Carballo JJ, Baca-García E, Artés Rodríguez A (2014) Objective diagnosis of ADHD using IMUs. Medical Engineering \& Physics 36: 922-926.

Poltavski DV, Biberdorf D, Petros TV (2012) Accommodative response and cortical activity during sustained attention. Vision Research 63:1-8.

Prado C, Dubois M, Valdois S. (2007) The eye movements of dyslexic children during reading and visual search: impact of the visual attention span. Vision Res 47: 2521-2530. 10.1016/j.visres.2007.06.001

Reh V, Schmidt M, Lam L, Schimmelmann BG, Hebebrand J, Rief W, Christiansen H. (2015) Behavioral Assessment of Core ADHD Symptoms Using the QbTest. J Atten Disord. (12):1034-45. doi: $10.1177 / 1087054712472981$

Riccio CA, Reynolds CR. (2001) Continuous performance tests are sensitive to ADHD in adults but lack specificity. A review and critique for differential diagnosis. Ann N Y Acad Sci. 931:113-39.

Ritsner MS, (2009) Editor. Neuropsychological Endophenotypes and Biomarkers: Springer Netherlands.

Rommelse NNJ, Van der Stigchel S, Sergeant JA. (2008) A review on eye movement studies in childhood and adolescent psychiatry, Brain and Cognition 68 (3): 391-414. 
Rouse MW, Borsting E, Hyman L, Hussein M, Cotter SA, Flynn M, Scheiman M, Gallaway M, De Land PN. (1999) Frequency of convergence insufficiency among fifth and sixth graders. The Convergence Insufficiency and Reading Study (CIRS) group. Optometry Vision Science. 76: 643-649.

Rouse M, Borsting E, Mitchell GL, Kulp MT, Scheiman M, Amster D, Coulter R, Fecho G, Gallaway M (2009). Academic behaviors in children with convergence insufficiency with and without parent-reported ADHD. Optometry Vision Science 86(10): 1169-1177.

Ross RG, Olincy A, Harris JG, Sullivan B, Radant A. (2000) Smooth pursuit eye movements in schizophrenia and attentional dysfunction: adults with schizophrenia, ADHD, and a normal comparison group. Biological Psychiatry, Volume 48, Issue 3, 197 - 203

Rothlind JC, Posner MI, and Schaughency EA (1991) Lateralized Control of Eye Movements in Attention Deficit Hyperactivity Disorder. Journal of Cognitive Neuroscience. 3:4, 377-381

Sangal RB, Sangal JM (2015) Use of EEG Beta-1 Power and Theta/Beta Ratio Over Broca's Area to confirm Diagnosis of Attention Deficit/Hyperactivity Disorder in Children. Clin EEG Neurosci. (3):177-82. doi: $10.1177 / 1550059414527284$.

Scassellati C, Bonvicini C, Faraone SV, Gennarelli M. (2012). Biomarkers and attention-deficit/hyperactivity disorder: a systematic review and meta-analyses. J Am Acad Child Adolesc Psychiatry 51(10):1003-19 e20.

Silk TJ, Vance A, Rinehart N, Bradshaw JL, Cunnington R. (2009) White-matter abnormalities in attention deficit hyperactivity disorder: a diffusion tensor imaging study. Hum Brain Mapp. 30(9):2757-65. 
Snyder SM, Rugino TA, Hornig M, Stein MA (2015) Integration of an EEG biomarker with a clinician's ADHD evaluation. Brain Behav. (4):e00330. doi: 10.1002/brb3.330.

Söderström S, Pettersson R, Nilsson KW (2014) Quantitative and subjective behavioural aspects in the assessment of attention-deficit hyperactivity disorder (ADHD) in adults. Nord J Psychiatry. 2014 Jan;68(1):30-7. doi: 10.3109/08039488.2012.762940.

Solé Puig M, Pérez Zapata L, Aznar Casanova JA, Supèr HA. (2013a) Role of eye vergence in covert attention. PloS One. 8(1): e52955. doi: 10.1371/journal.pone.0052955.

Solé Puig M, Puigcerver L, Aznar-Casanova JA, Supèr H (2013b) Difference in Visual Processing Assessed by Eye Vergence Movements. PLoS ONE 8(9): e72041. doi:10.1371/journal.pone.0072041

Solé Puig M, Pérez Zapata L, Puigcerver L, Esperalba Iglesias N, Sanchez Garcia C, Romeo A, et al. (2015) Attention-Related Eye Vergence Measured in Children with Attention Deficit Hyperactivity Disorder. PLoS ONE 10(12): e0145281. doi:10.1371/journal.pone.0145281

Soliva JC. (2011) Neuroimaging in the diagnosis of ADHD: where we are and where we are going. Expert Opinion Medical Diagnosis 5(4): 307-318.

Supèr H. Marco J, Zapata JP, Crespillo JC, Puig MS. (2014) Role of vergence during eye fixation in orienting visual attention. Journal of Vision 14(10), 9.

Suzuki S, Suzuki Y, Ohtsuka K (2004) Convergence eye movements evoked by microstimulation of the rostral superior colliculus in the cat. Neurosci Res. 49:39-45. 
Thome J, Ehlis AC, Fallgatter AJ, Krauel K, Lange KW, Riederer P, et al. (2012) Biomarkers for attentiondeficit/hyperactivity disorder (ADHD). A consensus report of the WFSBP task force on biological markers and the World Federation of ADHD. World J Biol Psychiatry. 13(5):379-400.

Thompson KG, Biscoe KL, Sato TR. (2005) Neuronal basis of covert spatial attention in the frontal eye field. Journal Neuroscience 25: 9479-9487.

Valera EM, Faraone SV, Murray KE, Seidman L. (2007) Meta-analysis of structural imaging findings in attention-deficit/hyperactivity disorder. Biological Psychiatry 61: 1361-1369.

Versino M, Hurko O, Zee DS (1996) Disorders of binocular control of eye movements in patients with cerebellar dysfunction. Brain 119:1933-1950.

Visser SN, Danielson ML, Bitsko RH, Holbrook JR, Kogan MD, Ghandour RM, et al. (2014) Trends in the parent-report of health care provider-diagnosed and medicated attention-deficit/hyperactivity disorder: United States, 2003-2011. J Am Acad Child Adolesc Psychiatry 53(1):34-46 e2. 


\section{Figure legends}

Figure 1

Schematic explanation of the vergence. The eyes focus on a single point in space where the angle of vergence $(\alpha)$ is formed by the two gaze lines.

Figure 2

Flowchart of the clinical validation.

Figure 3

Task design and images used. A: Illustration of the different phases of the attention task and stimulus presentation times. B: Gaze directions of the central frog act as a cue left or right. The target is an image of a tadpole and distractor an image of a fish.

Figure 4

Average modulation of the angle of vergence. Blue traces denote average angle of eye vergence from healthy control subjects. Green and red traces represent the vergence responses from Clinical control and ADHD subjects, respectively. Phases in the task are demarcated by vertical lines (Fix. denotes fixation period, Cue the cue period and Stimulus the period of target presentation). The lower dots indicate the time points when vergence responses significant $(p>0,05)$ differ between healthy and ADHD subjects (blue dots) and Clinical controls and ADHD subjects (green dots).

\section{Figure 5}

Average modulation of the angle of vergence. Blue and red traces denote average angle of eye vergence from cue and no-cue conditions. Labeling as in figure 4. 
Figure 6

Average modulation of the angle of vergence for distractor and target stimuli. Labeling as in figure 4.

Figure 7

ROC curves based on eye vergence.

Figure 8

Average modulation of pupil diameter. Blue traces denote pupil responses from healthy control subjects.

Green and red traces represent the pupil responses from Clinical control and ADHD subjects, respectively. Labeling as in figure 4.

Figure 9

ROC curves based on pupil size

Figure 10

Average modulation of the angle of vergence separated by behavioral response outcomes. Labeling as in figure 4.

Figure 11

Average modulation of the angle of vergence aligned on behavioral responses onset, i.e. the moment of button press. Labeling as in figure 4.

\section{Figure 12}

Average modulation of the angle of vergence according to task period. Blue and red traces denote average angle of eye vergence from cue and no-cue conditions. Labeling as in figure 4. 
Figure 13

Average modulation of the angle of vergence according to stimulus side. Blue and red traces denote average angle of eye vergence from cue and no-cue conditions. Labeling as in figure 4. 


$$
\alpha
$$


CONTROL SUBJECTS $(\mathrm{N}=51)$

without attentional or conduct problems
REFERRALS TO HOSPITAL ( $\mathrm{N}=108)$ with attentional or conduct problems
BGAZE ADHD TESTING

\section{BGAZE CLASSIFIER LAYER I}

- Controls: $\mathrm{N}=30 ;$ ADHD: $\mathrm{N}=23$

- Testing, training \& cross-validation

- Accuracy 96\%

- Not blinded

BGAZE CLASSIFIER LAYER II

- Clinical controls: $\mathrm{N}=19 ;$ ADHD: $\mathrm{N}=20$

- Testing, training \& cross-validation

- Accuracy: 88\%

- Not blinded

\section{'GOLD STANDARD' CLINICAL DIAGNOSIS} Blinded

\section{CLINICAL EVALUATION}
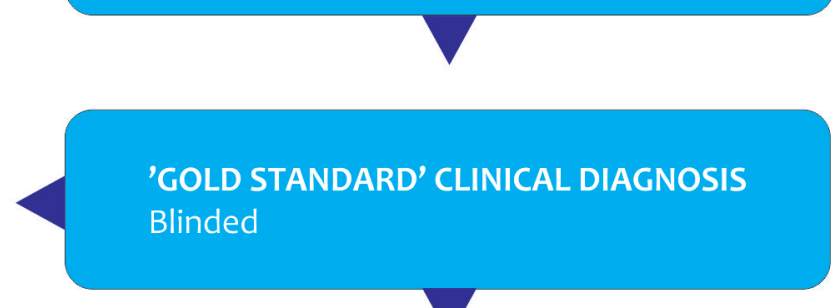

\section{$\checkmark$}

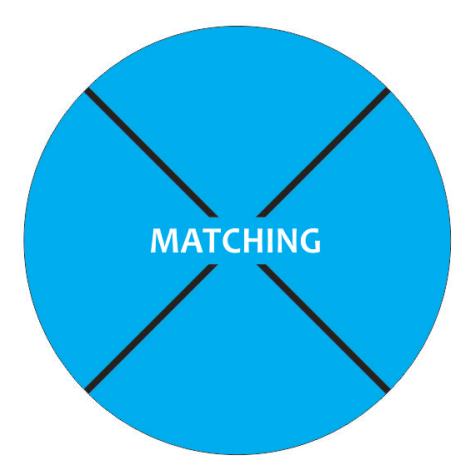

\section{BGAZE VALIDATION}

- Controls: N=21; Clinical controls: 14; ADHD: N=32 - Accuracy Controls -ADHD: 96,3\%

- Accuracy Clinical controls - ADHD: 85,7\% - Blinded 


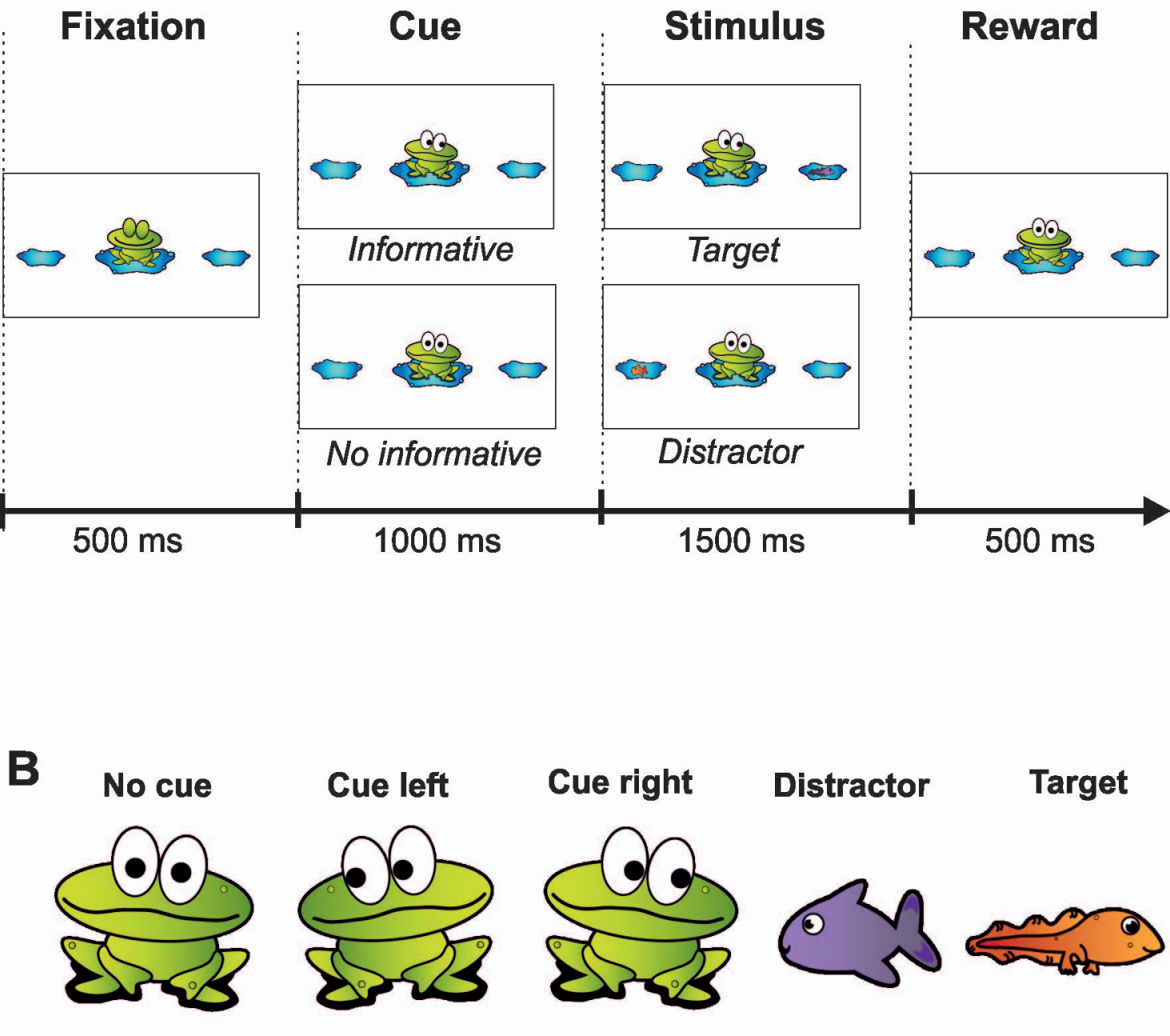




\section{No informative cue}

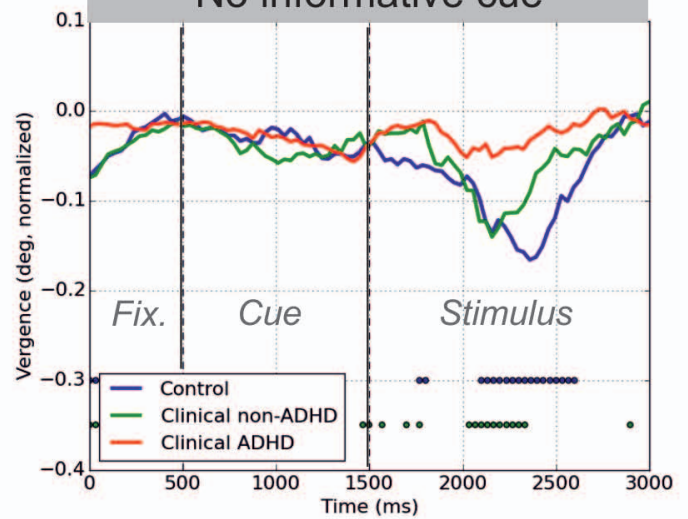

Informative cue

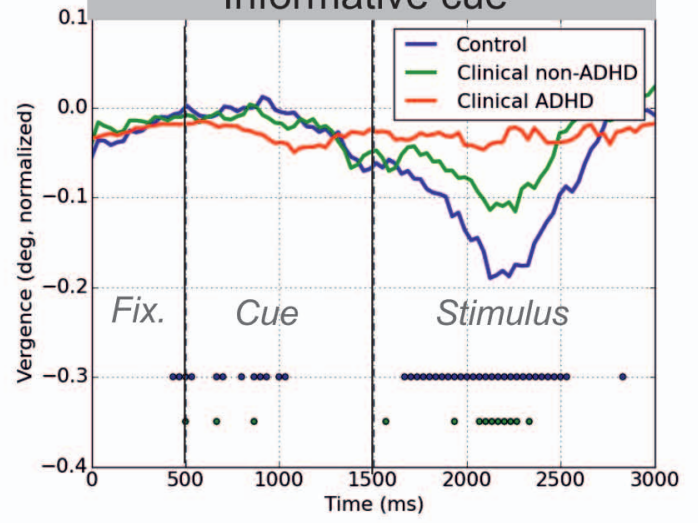


Control

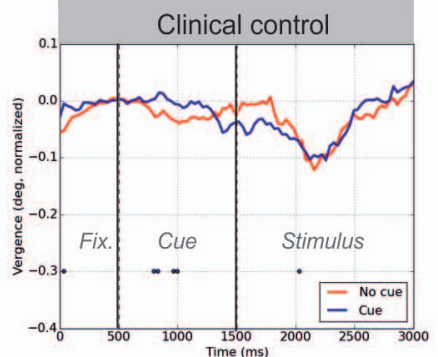




\section{Distractor}

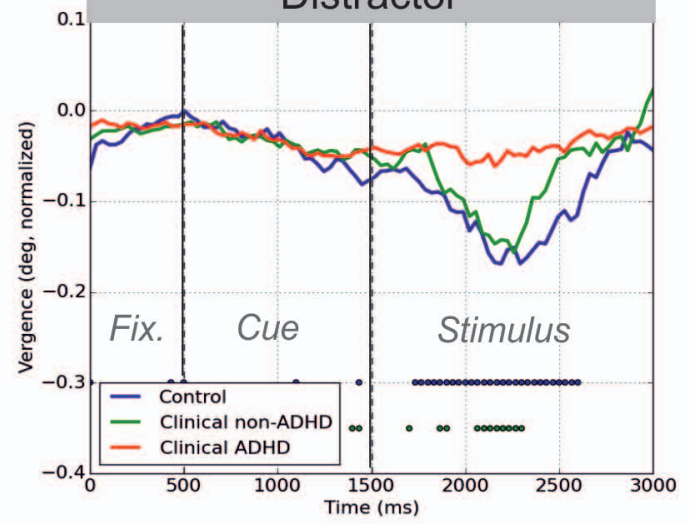

Target
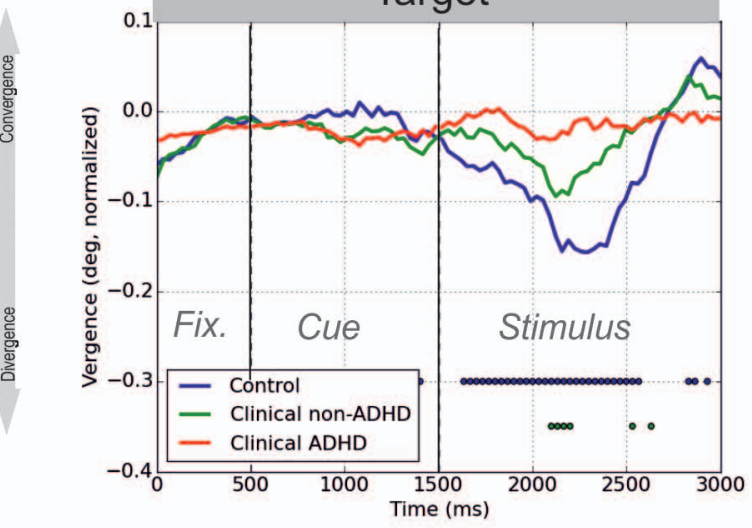


\section{Control vs ADHD}

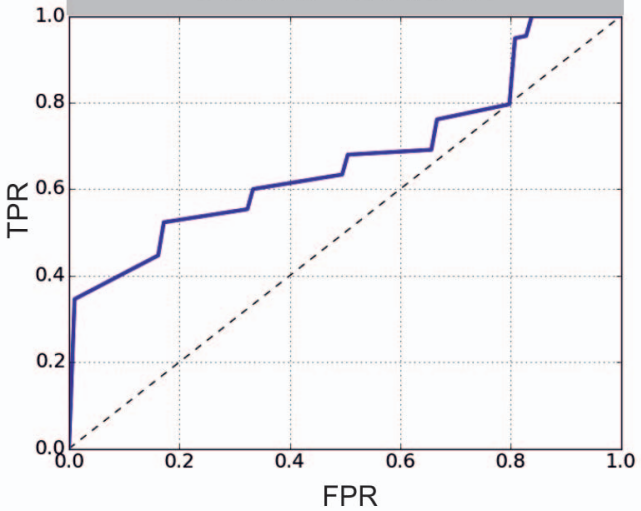




\section{No informative cue}
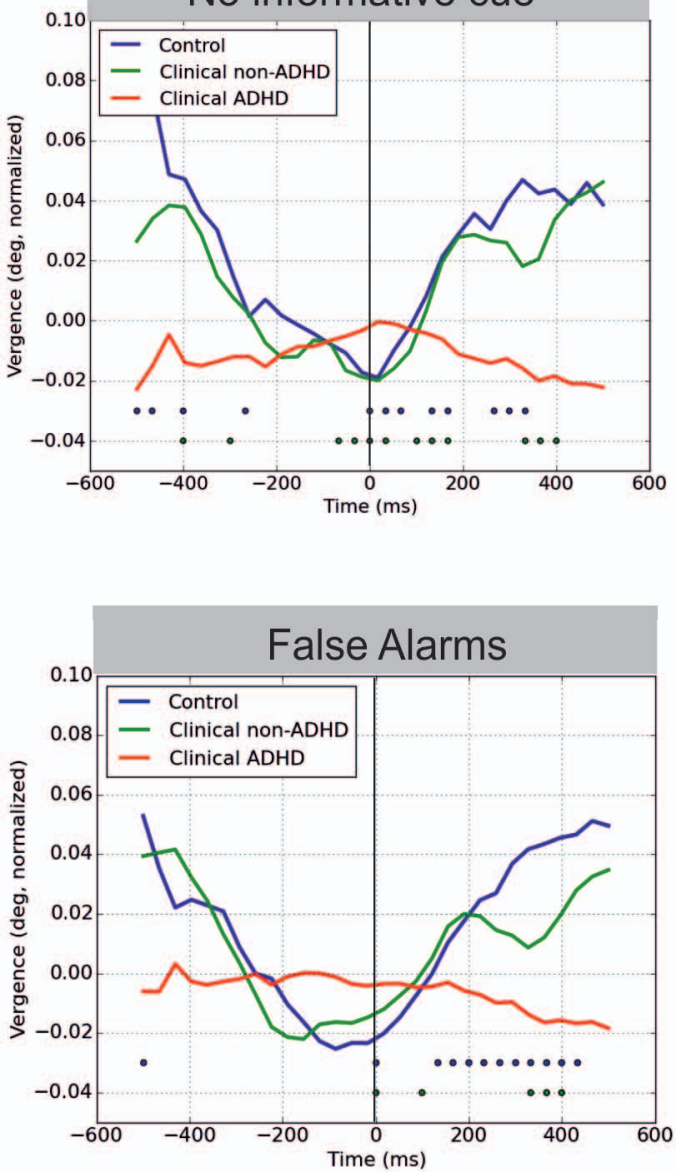

Informative cue

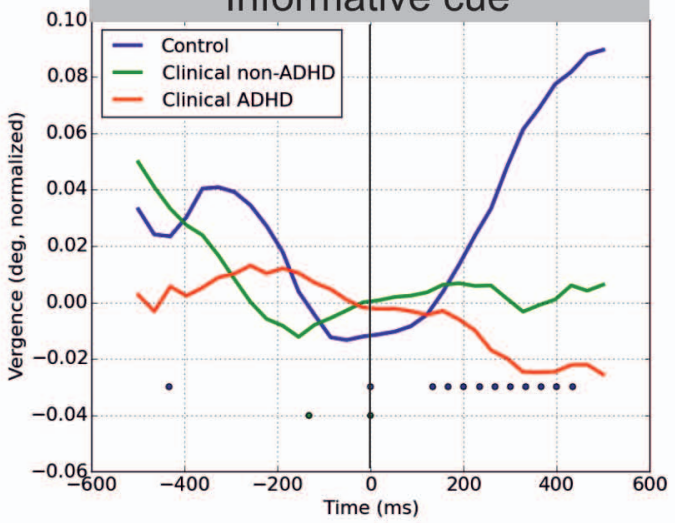

Hits

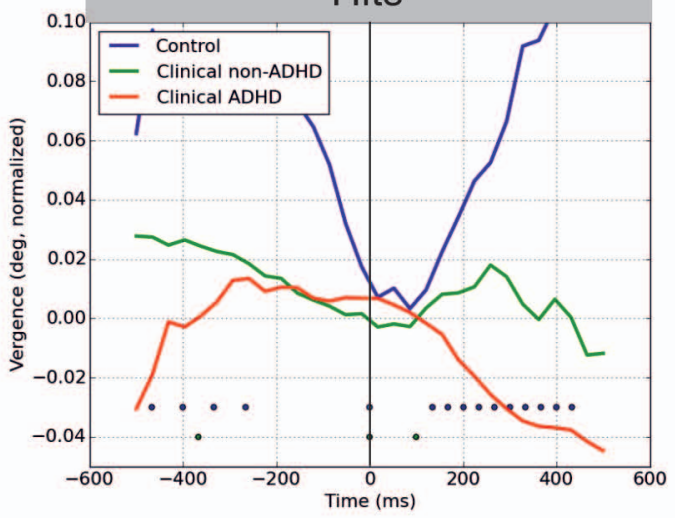




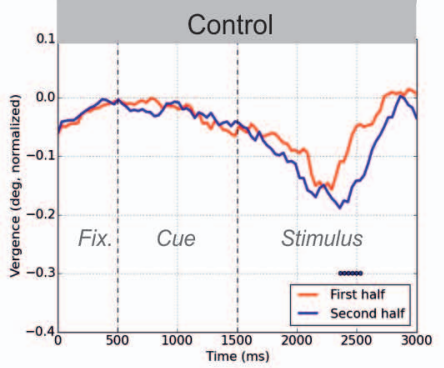

\section{Clinical control}

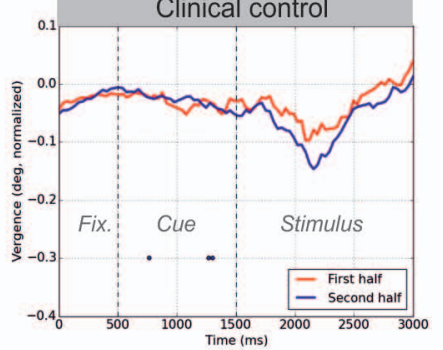

ADHD

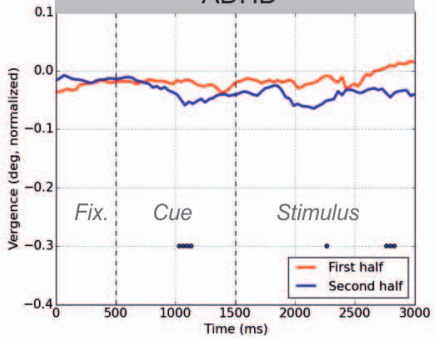




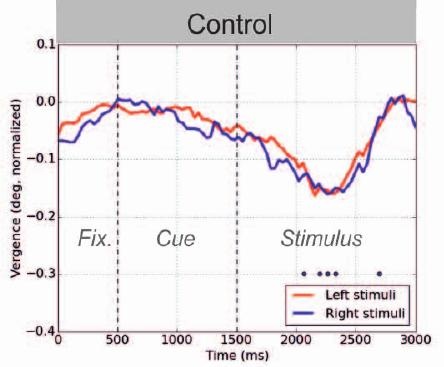

\section{Clinical control}

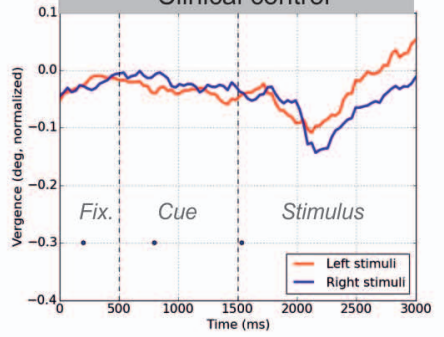

ADHD

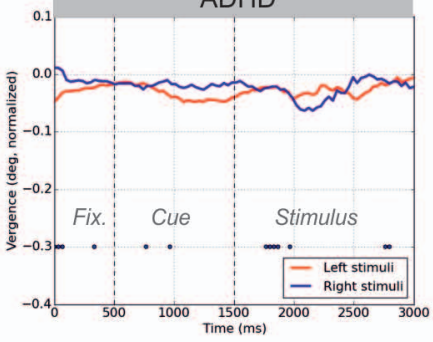

